\title{
étude de l'activité sismo-acoustique liée à une injection de fluide dans un massif granitique
}

\author{
S. TALEBI et F.H. CORNET \\ Laboratoire de sismologie \\ Institut de Physique du Globe de Paris*
}

\section{Résumé}

L'injection de $6 \mathrm{~m}^{3}$ de gel à $200 \mathrm{~m}$ de profondeur dans un massif granitique a provoqué une abondante activité sismo-acoustique dans le domaine $0,4-5 \mathrm{kHz}$. Le débit d'injection a varié de 180 à $520 \mathrm{l} / \mathrm{min}$, la pression maximum en tête de forage atteignant $12 \mathrm{MPa}$.

Ces événements ont été localisés à partir des lectures des temps d'arrivée des ondes $\mathrm{P}$ et $\mathrm{S}$ observées sur quatre sondes trois composantes mises en place, dans deux forages, au voisinage du point d'injection. Ils se distribuent dans une zone sub-horizontale; la distance moyenne des événements vis-à-vis du point d'injection augmentant régulièrement au cours du temps. Ces résultats suggèrent que la fracture hydraulique sub-verticale identifiée au niveau du forage n'a eu qu'une extension limitée, l'écoulement loin du forage intervenant dans une zone perméable préexistante.

L'analyse spectrale des signaux observés met en évidence des fréquences-coin de l'ordre de $1200 \mathrm{~Hz}$ et des moments sismiques de l'ordre de 11000 N.m. Ces valeurs sont cohérentes avec des ruptures par cisaillement le long de surfaces planes circulaires de rayon moyen de l'ordre de $0,9 \mathrm{~m}$ associées à des chutes de contrainte moyenne égales à $5 \mathrm{kPa}$, valeurs obtenues sans tenir compte des effets de l'atténuation.

D'après les mesures de contrainte régionale et l'étude de la fracturation naturelle du massif, ces mouvements auraient été induits par des augmentations de pression interstitielle supérieures ou égales à $3,8 \mathrm{MPa}$. Ces microglissements ne seraient pas intervenus dans le domaine de forte conductivité hydraulique dans lequel a eu lieu l'écoulement mais dans les épontes de ce dernier.

\section{Abstract}

The injection of $6 \mathrm{~m}^{3}$ of gel at a depth of $200 \mathrm{~m}$ in a granite rock mass generated numerous seismo-acoustic events in the band $0.4-5 \mathrm{kHz}$ frequency domain. During injection, the flow rate changed from 180 to $520 \mathrm{l} / \mathrm{min}$; the pressure at the borehole head reached $12 \mathrm{MPa}$.

These events were located by using $P$ and $S$ wave arrival times on four 3components packages anchored in two adjacent boreholes near the injection point. They are distributed in a sub-horizontal area and progressively migrated away from the injection well as the time passed. These results suggest that the hydraulic subvertical fracture identified at the borehole wall had a limited extension in space and that flow occured through a preexisting permeable zone away from the wellbore.

The spectral analysis of these signals shows corner frequencies of the order of $1200 \mathrm{~Hz}$ and seismic moments of about $11000 \mathrm{~N} . \mathrm{m}$. This is coherent with failures along penny shaped cracks of radii equal to about $0.9 \mathrm{~m}$ and mean stress drops about $5 \mathrm{kPa}$. These values have been obtained without corrections of attenuation effects.

According to the regional stress determination and to local natural fracture pattern. these movements may have been triggered by an increase of pore pressure of about 3.8 MPa. These shear events seem to have occured along natural fractures intersecting the preexisting sub-horizontal high conductivity zone but not within the zone itself.

\footnotetext{
* Université Pierre-et-Marie-Curie, 4, place Jussieu, 75230 Paris cedex 5.
} 


\section{INTRODUCTION}

Depuis plusieurs années des efforts importants ont été entrepris pour essayer de comprendre la manière dont s'effectue l'écoulement d'un fluide dans un massif rocheux en étudiant l'activité sismo-acoustique engendrée par des variations de pression de fluide (POWER et al., 1975; ALBRIGHT ET PEARSON, 1980; PEARSON, 1981; LEYDECKER, 1981). Dans tous les cas il s'agit tout d'abord de déterminer le domaine dans lequel des variations de pression sont intervenues puis, si possible, d'identifier la source des événements observés: ruptures par traction associées à des fractures hydrauliques ou ruptures par cisaillement associées à des variations de contrainte effective.

Le propos de l'étude décrite ci-après effectuée dans le massif granitique du Mayet-de-Montagne situé à $25 \mathrm{~km}$ au sud-est de Vichy était d'observer dans un large domaine de fréquences l'activité sismo-acoustique associée à une injection de gel, en disposant de capteurs le plus près possible du point d'injection. L'essai d'injection proprement dit était destiné à étudier la possibilité de développer une fracture hydraulique dans une direction qui ne soit pas perpendiculaire à la direction de la contrainte principale minimum locale, la cartographie de l'activité sismo-acoustique devant permettre de préciser la géométrie de la fracture loin du forage.

\section{DESCRIPTION DU SYSTÈME D'ÉCOUTE}

\section{Les sondes sismo-acoustiques}

Les sondes utilisées ont été conçues par la société allemande Prakla-Seismos et testées sur le site de Falkenberg en R.F.A. (LEYDECKER, 1981).

Trois accéléromètres, piezo-électriques de fréquence propre comprise entre 4 et $5 \mathrm{kHz}$, mais linéaires dans le domaine $0-3 \mathrm{kHz}$ sont installés selon trois axes orthogonaux, au centre de chaque sonde. La sensibilité moyenne de ces capteurs est de 5 volt $/ \mathrm{g}(1 \mathrm{~g}=9,81 \mathrm{~m} / \mathrm{s})$; le bruit électronique avant amplification est d'environ 4 micro-volts. L'orientation des accéléromètres horizontaux est connue grâce à une boussole incorporée à la sonde.

Un ensemble de quatre sondes a été mis en place dans deux forages à des cotes variant de $135 \mathrm{~m}$ à $192 \mathrm{~m}$ (fig. 1). Chaque sonde est descendue à la cote requise puis fixée contre la roche par un bras d'ancrage mis en mouvement par un moteur interne à la sonde. Le signal reçu est amplifié de $56 \mathrm{db}$ avant d'être transmis à la surface, cette amplification étant constante pendant toute l'expérience.

Les sondes $A$ et $B$ se trouvaient dans le forage INAG 3-2 tandis que les sondes $C$ et $D$ se trouvaient dans le forage INAG 3-3. Ces deux forages sont situés aux sommets d'un triangle équilatéral de $30 \mathrm{~m}$ de côté, le troisième sommet étant occupé par le forage d'injection.

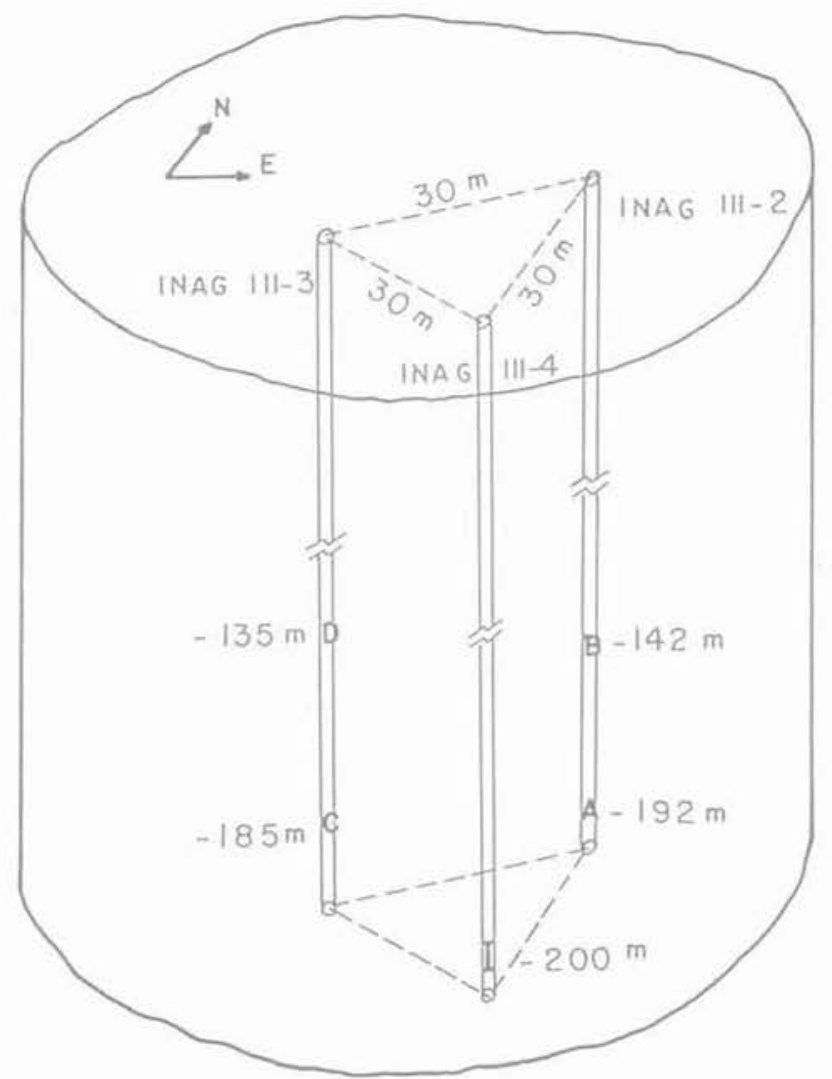

Fig. 1. - Dispositif d'écoute sismique du Mayet-deMontagne. Le point I dans le forage INAG III-4 correspond au point d'injection, tandis que les sondes occupaient les points $A, B, C$ et $D$ dans les forages INAG III-2 et INAG III-3.

Nous mentionnerons également pour mémoire qu'une sonde trois composantes, de bande passante $10 \mathrm{~Hz}$ $250 \mathrm{~Hz}$, avait été installée à $50 \mathrm{~m}$ de profondeur dans un forage situé à $20 \mathrm{~m}$ du forage d'injection, mais aucun signal n'ayant été enregistré par cette sonde, il n'y sera plus fait référence par la suite. Cette absence de signal sera expliquée lors de la discussion sur le contenu spectral des signaux.

\section{Le système d'acquisition des données}

Les signaux émis par les sondes ont été enregistrés en permanence pendant la durée totale de l'injection sur un enregistreur magnétique à seize pistes après qu'ils eurent été amplifiés. Le gain des amplificateurs, variables de $-40 \mathrm{db}$ à $+20 \mathrm{db}$ était ajusté en permanence en fonction du son produit par quatre hautparleurs reproduisant le niveau sonore des signaux.

La vitesse de déroulement de la bande magnétique était de $4,75 \mathrm{~cm} / \mathrm{s}$ assurant une bande passante de $0-7 \mathrm{kHz}$, En plus de l'enregistrement en mode FM des douze signaux provenant des sondes, un code horaire et un signal de fréquence stable égale à $1000 \mathrm{~Hz}$ ont êté enregistrés. Ce dernier avait pour but d'assurer rigoureusement la même vitesse de déroulement durant l'enregistrement et durant les rejeux. 
En outre, les signaux étaient tracés en permanence sur un traceur à douze pistes présentant une bande passante de 0 à $5 \mathrm{kHz}$ et déroulant son papier (sensible aux ultra-violets) à $1 \mathrm{~cm} / \mathrm{s}$. Ces traces permettaient un contrôle continu du bon fonctionnement des sondes,

Après l'acquisition de ces données sur le terrain, les signaux ont été numérisés après avoir été filtrés en «passe-bande» dans le domaine $0-5 \mathrm{kHz}$. Le pas d'échantillonnage de chaque trace était de 70 microsecondes, chaque valeur étant codée en mots de douze bits.

\section{L'ESSAI D'INJECTION}

L'essai d'injection a été réalisé à l'aide d'un double obturateur gonflable situé à $197 \mathrm{~m}$ de profondeur dans le forage INAG 3-4 (fig. 1). Cette cote avait été choisie après qu'une étude par caméra de forage eut révélé la présence à cette profondeur d'une veine de quartz, ou de calcite, sub-verticale dans la direction $\mathrm{N} 155^{\circ} \mathrm{E}$, la contrainte principale maximum horizontale étant, elle, orientée $\mathrm{N} 19^{\circ} \mathrm{E}$ (CORNET et VALETTE, 1984).

L'essai d'injection s'est déroulé en trois étapes. Tout d'abord une injection d'eau à très faible débit (1 1/min) d'une vingtaine de litres a été réalisée pour essayer de réouvrir la veine de calcite. On admettait que la résistance à la traction de cette dernière était beaucoup plus faible que celle de la roche saine. Aucune écoute sismo-acoustique n'a été faite durant cette opération.

Dans un deuxième temps, du gel de viscosité égale à environ 300 centipoises a été injecté tout d'abord au débit de $180 \mathrm{l} / \mathrm{min}$ puis, après deux minutes, à $520 \mathrm{l} / \mathrm{min}$ pour une pression en tête de forage de $12 \mathrm{MPa}$. Du sable a alors été ajouté au gel pour permettre de maintenir la fracture ouverte à la fin de l'injection.

Malheureusement, le système s'est obstrué alors qu'environ $300 \mathrm{~kg}$ de sable avait été injecté. Après nettoyage du train de tige, l'injection a été reprise, avec du gel seulement, à un débit de $520 \mathrm{l} / \mathrm{min}$. Cette injection a dô être à nouveau interrompue du fait de l'apparition d'un court-circuit. Une production de plus en plus abondante d'eau claire d'abord, de gel ensuite, est en effet intervenue dans l'annulaire. Un total de $6 \mathrm{~m}^{3}$ de gel avait alors été injecté.

La figure 2 montre la variation en fonction du temps de la pression en tête de forage et la variation du niveau d'eau dans les forages III- 2 et III- 3 lors de la deuxième étape. On remarque que la montếe du niveau d'eau dans le forage III-3 intervient quelques minutes après le début de l'injection, tandis que le niveau d'eau dans le forage III-2 reste relativement stable. Ceci suggère que le gel s'est dirigé vers III-3 plutôt que vers III-2.

A la fin des essais, une étude par vidéo-carottage a indiqué qu'une fracture sub-verticale avait effectivement été créée dans la direction $\mathrm{N} 155^{\circ} \mathrm{E}$, c'est-à-dire dans la direction du filonnet de quartz ou de calcite identifié avant les essais.

Durant la deuxième injection de nombreux événements sismo-acoustiques ont été observés dont un exemple est présenté sur la figure 3 . On a reporté sur la figure 2 le nombre des événements dépassant une amplitude de \pm 1 volt (amplifié de $70 \mathrm{db}$ ) lors de l'enregistrement magnétique (ce quí correspond à une accélération de $63 \mu \mathrm{g}$ ).

Très peu d'événements ont été observés lors de la troisième injection. Les résultats décrits ci-après portent donc sur les signaux observés lors de la deuxième injection.

\section{LOCALISATION DES ÉVÉNEMENTS}

\section{Méthode employée}

La localisation des événéments consiste à déterminer les coordonnées $\mathrm{X}, \mathrm{Y}, \mathrm{Z}$, des foyers et le temps t où l'événement est intervenu. La précision de la détermination dépend du nombre de données disponibles, un minimum de quatre valeurs indépendantes étant indispensable.

Cette localisation a été obtenue par une méthode itérative basée sur la détermination des temps d'arrivée des ondes $\mathrm{P}$ et $\mathrm{S}$. A partir des lectures des temps d'arrivée, on choisit a priori un foyer et un temps approximatif pour un événement donné. Soit $x_{0}, y_{0}$ et $\mathrm{z}_{0}$ les coordonnées de ce point et $t_{0}$ le temps d'apparition de cet événément. Ce choix conduit à calculer l'intervalle de temps $T_{\text {, }}$ requis pour que le signal parvienne au capteur $\mathrm{i}\left(\mathrm{d}_{1} / v_{\mathrm{p}}\right.$ pour l'onde $\mathrm{P}$ et $\mathrm{d} / \mathrm{V}_{\mathrm{s}}$ pour l'onde $\mathrm{S}$, où $\mathrm{d}_{\mathrm{i}}$ est la distance sourcecapteur $i, v_{p}$ et $v_{s}$ sont les vitesses des ondes $P$ et $S$ dans le milieu). L'erreur entre le temps observé t et le temps calculé $t_{0}+T_{1}$ est donc:

$$
F_{1}=t_{1}-\left[t_{0}+T_{1}\left(x_{0}, y_{0}, z_{0}\right)\right]
$$

On cherche alors la solution $x, y, z, t$ qui minimise la somme des carrés, des erreurs $\mathrm{F}_{1}$. Il faut noter que la qualité de la détermination dépend non seulement du nombre et de la précision des lectures des temps d'arrivée des ondes $\mathrm{P}$ et $\mathrm{S}$, mais aussi de la connaissance des vitesses de ces ondes dans le milieu.

\section{Détermination des vitesses des ondes $\mathrm{P}$ et $\mathrm{S}$}

Les vitesses des ondes $\mathrm{P}$ et $\mathrm{S}$ ont été mesurées sur le terrain de deux manières différentes: à partir d'une diagraphie sonique pour la vitesse des ondes $\mathrm{P}$ et à partir de l'enregistrement des signaux créés par des tirs de dynamite pour les vitesses des ondes $\mathrm{P}$ et $\mathrm{S}$.

La diagraphie sonique consiste à mesurer le temps de passage d'une impulsion $(15-25 \mathrm{kHz})$ entre deux récepteurs distants de $50 \mathrm{~cm}$ l'un de lautre. Cette 

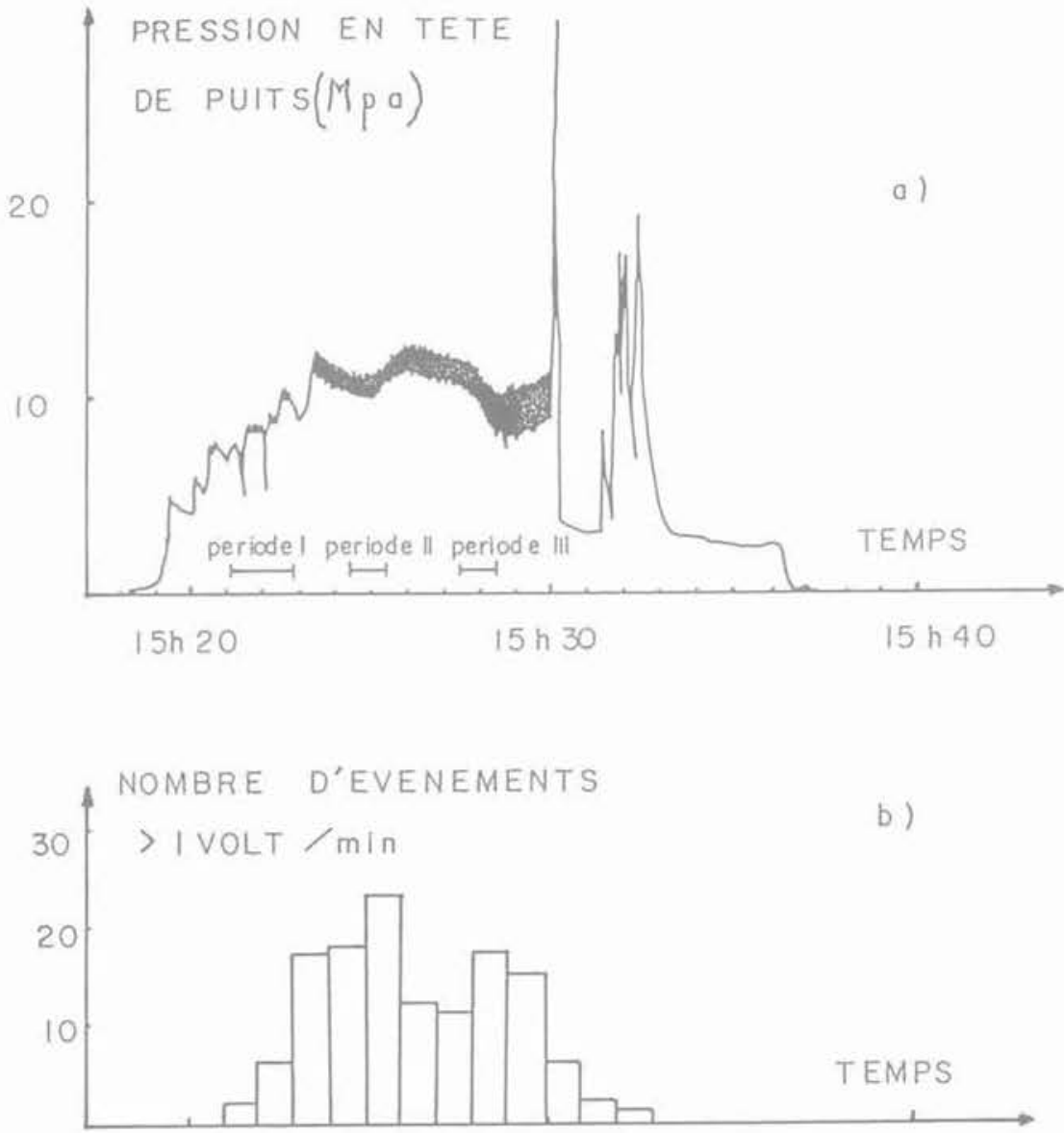

$15 \mathrm{~h} 2 \mathrm{O}$

$15 \mathrm{~h} 30$

15 h 40

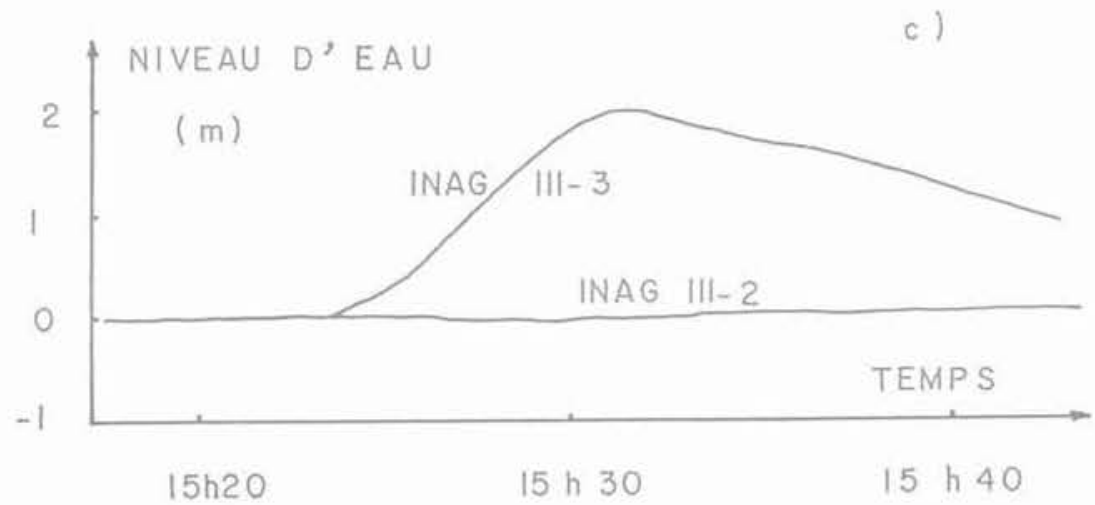

Fig. 2. - Variation en fonction du temps; a) de la pression d'injection, bl du nombre d'événements dépassant une amplitude de \pm 1 volt sur la composante H2 de la sonde $B$, et c) du niveau d'eau dans les forages INAG III-2 et INAG III-3. 


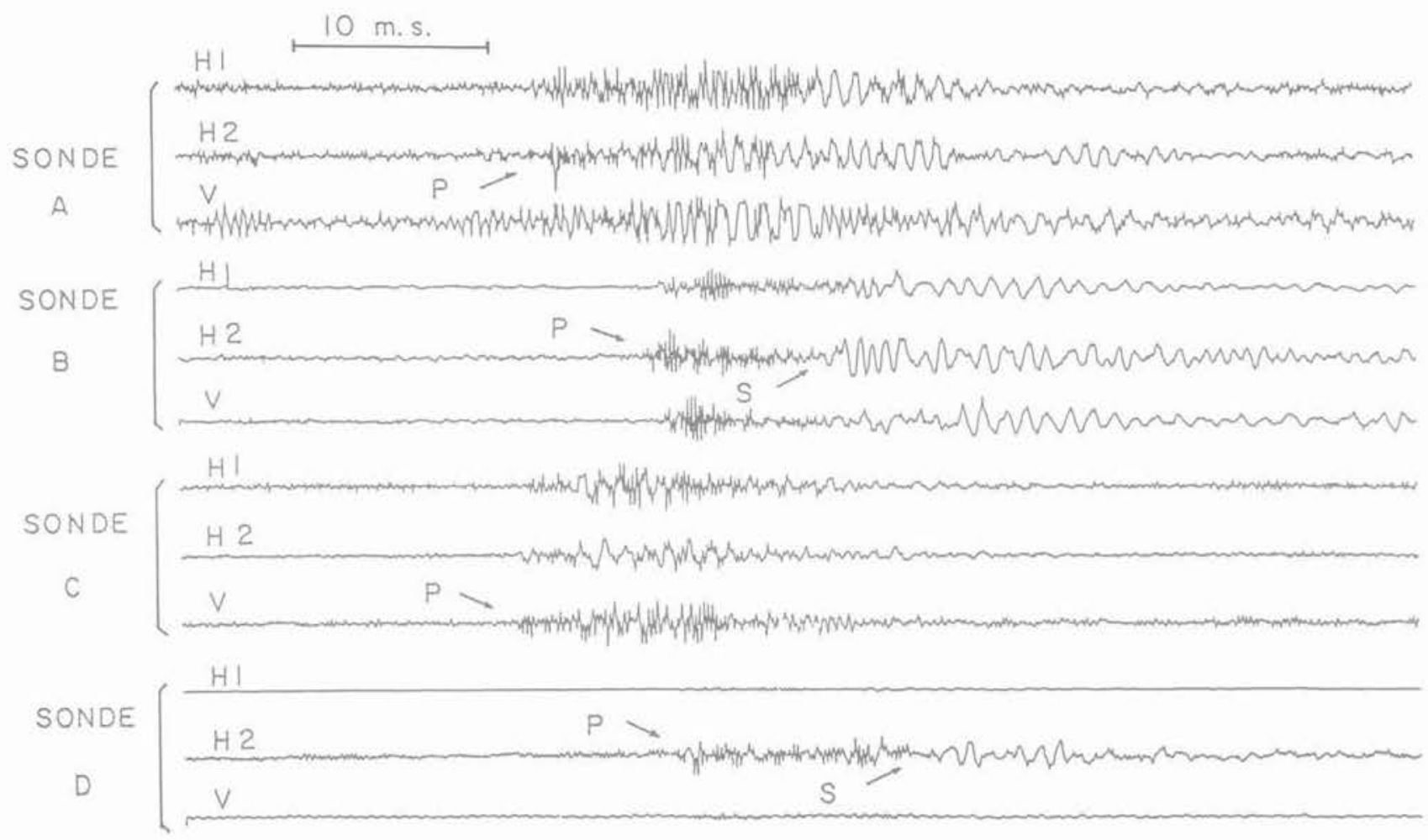

Fig. 3. - Exemple d'un événement sismo-acoustique.

opération, réalisée sur toute la profondeur du puits III-4, donne des vitesses relativement stables d'une valeur moyenne de $5600 \mathrm{~m} / \mathrm{s}$ pour l'onde $P$, entre 10 et $220 \mathrm{~m}$ de profondeur.

Les vitesses déterminées à partir des tirs de dynamite à différentes profondeurs pour des distances sourcecapteur de 10 à $120 \mathrm{~m}$ et pour des directions azimutales couvrant un secteur de $200^{\circ}$ varient de $5800 \mathrm{~m} / \mathrm{s}$ pour la direction $\mathrm{N} 145^{\circ} \mathrm{E}$ à $5300 \mathrm{~m} / \mathrm{s}$ pour une direction perpendiculaire (AUDRIC, 1980). Les valeurs moyennes retenues sont de $5650 \mathrm{~m} / \mathrm{s}$ pour la vitesse des ondes $P$ et de $3200 \mathrm{~m} / \mathrm{s}$ pour la vitesse des ondes $\mathrm{S}$.

\section{Les hypocentres des événements}

Pour la majorité des signaux enregistrés, l'arrivée des ondes $\mathrm{P}$ et $\mathrm{S}$ est assez nette comme le montre l'exemple de la figure 3 . Seuls les composantes $\mathrm{H}_{1}$ et $\mathrm{V}$ de la sonde $\mathrm{D}$ n'ont pas bien fonctionné au cours de l'essai. Cependant, la troisième composante de cette sonde a bien enregistré les phases $\mathrm{P}$ et $\mathrm{S}$ et a donc permis d'obtenir des lectures de temps d'arrivée.

Les événements enregistrés pendant trois périodes d'environ 1 à 2 minutes après 1,4 et 7 minutes de pompage ont été étudiés dans le but de mettre en évidence une éventuelle migration (fig. 2). Une vingtaine d'événements pour chacune de ces périodes présentent des arrivées d'ondes $\mathrm{P}$ et $\mathrm{S}$ suffisamment nettes pour permettre de les localiser à partir de cinq ou six lectures. Une méthode par moindres carrés a ensuite été utilisée pour définir le meilleur plan passant par chaque collection d'hypocentres. Les résultats ainsi obtenus sont résumés dans le tableau I, où les périodes 1,2 et 3 correspondent aux trois périodes de 1 à 2 minutes mentionnées précédemment et la période 4 correspond à l'ensemble des événements étudiés pour ces trois périodes auxquels nous avons ajouté quelques événements supplémentaires. L'examen des résultats du tableau 1 conduit à quelques observations concernant la distribution des événements dans le massif, ainsi que la forme géométrique de cette distribution.

Tableau 1. - Résultats des déterminations des meilleurs plans passant par les hypocentres des événements. NE est le nombre d'événements étudiés; $\mathrm{Dm}$ est la distance moyenne des événements visà-vis du point d'injection; l'azimut et le pendage indiqués correspondent au meilleur plan passant par les hypocentres des événements

\begin{tabular}{|c|c|c|c|c|c|}
\hline Période & NE & $\begin{array}{l}\mathrm{Dm} \\
(\mathrm{m})\end{array}$ & Azimut & $\begin{array}{l}\text { Pen- } \\
\text { dage }\end{array}$ & $\begin{array}{c}\text { Ecart- } \\
\text { type } \\
\text { (m) }\end{array}$ \\
\hline 1 & 20 & 10,7 & $\mathrm{~N} 93 \mathrm{E}$ & $31^{\circ}$ & 2,5 \\
\hline 2 & 19 & 12,0 & $\mathrm{~N} \quad 69 \mathrm{E}$ & $17^{\circ}$ & 21 \\
\hline 3 & 19 & 16,9 & $\mathrm{~N} 150 \mathrm{E}$ & $14^{\circ}$ & 4,0 \\
\hline 4 & 63 & 13,2 & $\mathrm{~N} 113 \mathrm{E}$ & $21^{\circ}$ & 3,5 \\
\hline
\end{tabular}

La distance moyenne entre le point d'injection et les événements pour les périodes 1,2 et 3 est respectivement de $10,7,12,0$ et $16,9 \mathrm{~m}$, ce qui montre clairement l'éloignement progressif des hypocentres visà-vis du point d'injection. Ce résultat est cohérent avec une diffusion progressive de l'eau dans le massif au cours du temps. D'autre part, les plans déterminés 
par moindres carrés à partir des hypocentres des événements sont de faible pendage et ce d'autant plus que les événements s'éloignent du point d'injection. On remarquera que les écarts-types des distances des événements vis-à-vis de ces plans sont suffisamment petits pour qu'il soit permis de conclure que la perturbation de la pression interstitielle du fait de l'injection est intervenue essentiellement dans une zone sub-horizontale. Cependant l'écart-type pour la période étudiée après sept minutes de pompage est environ double de celui pour la période après quatre minutes, ce qui serait cohérent avec une percolation progressive du fluide dans le massif à partir des épontes de la zone hydrauliquement conductrice.

Nous avons représenté sur la figure 4 la projection dans un plan horizontal des événements pour ces différentes périodes. On y constate qu'à mesure que les événements s'éloignent du point d'injection, ils ont tendance à migrer vers le forage III-3 ce qui est en parfait accord avec l'observation de l'élévation du niveau d'eau dans ce forage. L'ordre chronologique d'occurrence des evénements et la distance de ces derniers vis-à-vis du point d'injection excluent qu'ils soient associés au front d'écoulement du gel mais indiquent qu'ils sont le résultat d'une augmentation de pression interstitielle dans une zone hydrauliquement conductrice préexistante (tab. I et fig. 4).

Ces résultats suggèrent donc que si une fracturation hydraulique du forage est bien intervenue au début des essais dans un plan vertical $N 155^{\circ} \mathrm{E}$, l'écoulement est intervenu par la suite dans une zone sub-horizontale.

\section{5. ÉTUDE DE LA SOURCE DES ÉVÉNEMENTS OBSERVÉS}

L'injection de fluides sous pression dans un massif rocheux peut engendrer des mouvements vibratoires, soit du fait de la propagation de fractures hydrauliques, soit du fait de micro-glissements le long de fractures préexistantes induites par l'accroissement de pression interstitielle. Ces deux mécanismes fournissent des * signatures * spectrales caractéristiques pour les vibrations qui leur sont associées ce qui permet a posteriori d'identifier le mécanisme à la source des événements observés.

\section{Caractéristiques spectrales des événements engendrés par une injection d'eau}

Les vibrations engendrées par la propagation d'une fracture hydraulique ont été étudiés par AKI et al., (1977) et CHOUET, (1981). Plus particulièrement AKl et al. ont montré que la propagation d'une telle fracture dans un milieu parfaitement homogène devrait être stable et ne devrait donc pas engendrer de vibration au cours de sa propagation. Cette conclusion est obtenue à partir du calcul du facteur d'intensité de contrainte en fond de fracture lorsque le fluide ne mouille pas la fracture jusqu'à son extrémité. Ils ont conclu de ce fait que la propagation d'une fracture hydraulique ne pouvait engendrer des vibrations que si la résistance à la traction du matériau était hétérogène, ou s'il existait une différence entre les valeurs

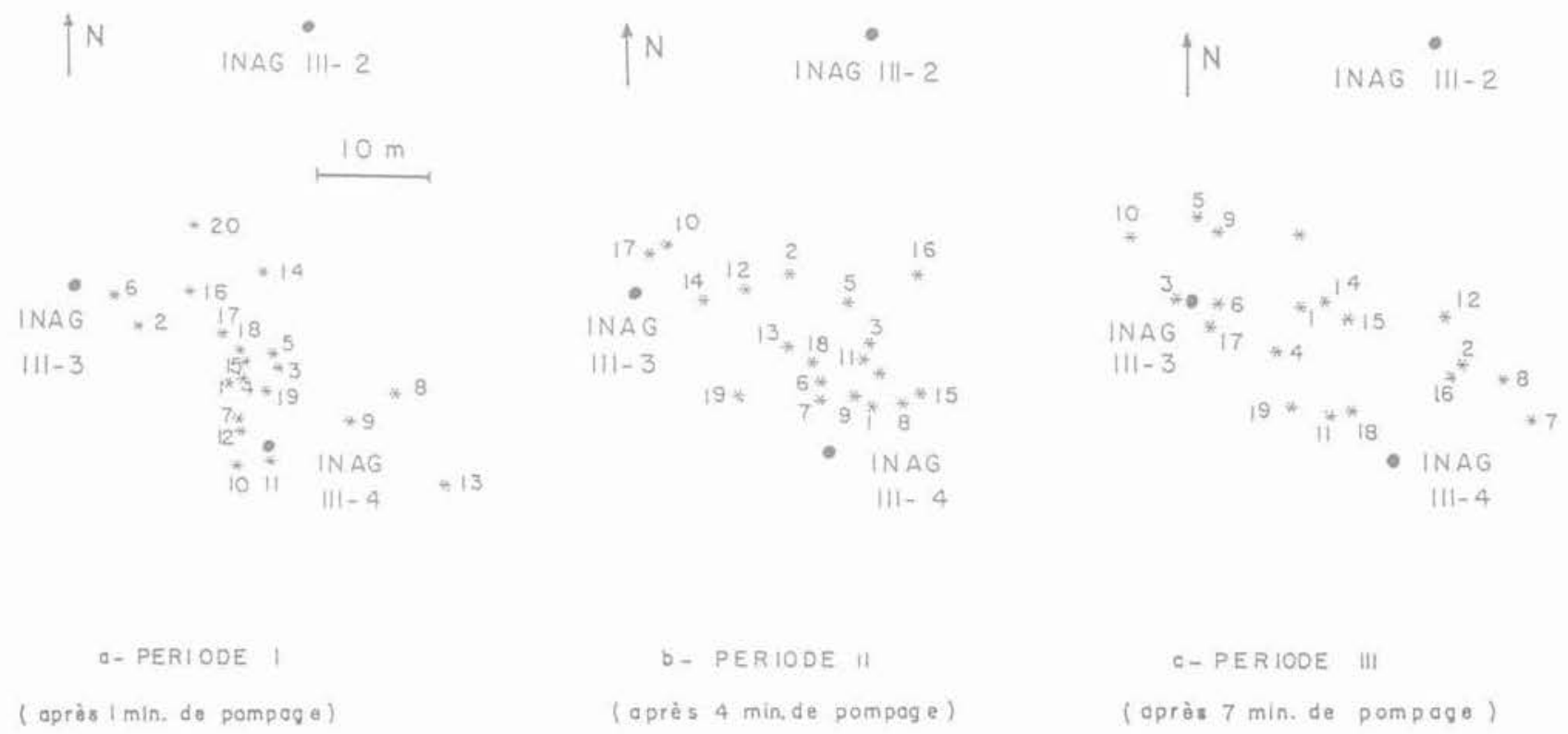

Fig. 4. - Projection sur le plan horizontal des hypocentres d'événements observés. Les numéros indiquent l'ordre d'apparition des événements pour chaque période. 
critiques statique et dynamique du facteur d'intensité de contrainte, induisant de ce fait un avancement saccadé du front de fracture.

Des résultats récents (RUMMEL, 1985) obtenus au laboratoire lors de l'observation de la propagation de fractures hydrauliques dans du plexiglass ont montré que ces fractures se propageaient de façon saccadée bien que le matériau soit parfaitement homogène.

Il est possible d'expliquer ce phénomène, nous semble$\mathrm{t}$-il, si l'on se rappelle que la propagation d'une fracture hydraulique est instable lorsque la pression est appliquée uniformément jusqu'au fond de la fracture (voir par exemple ZOBACK et POLLARD, 1977. à ce sujet). En effet, avant la propagation, le fluide mouille la fracture jusqu'au fond et lorsque la pression atteint une valeur critique, la fracture se propage à une vitesse supérieure à la vitesse d'écoulement du fluide ce qui induit une chute de pression. Selon la manière dont se fait l'écoulement du fluide en fond de fracture, c'est-à-dire selon les valeurs de la viscosité et de la compressibilité du fluide de fracturation, on conçoit qu'il est possible soit d'observer une nouvelle propagation instable soit une propagation stable.

Quel que soit le mécanisme invoqué, il semble maintenant acquis que des instabilités de propagation puissent intervenir, donnant soudainement naissance à des discontinuités dans le champ des déplacements dans la direction normale au plan de fracture. L'étude des vibrations associées à de telles sources a été menée par AKI et al. et CHOUET, ce qui les a conduit à conclure que ces instabilités donnaient naissance à des ondes dont l'énergie est centrée autour d'une ou plusieurs fréquences dont les valeurs moyennes dépendent de la longueur totale de la fracture.

Rappelons enfin que d'après SAVAGE and MANSINHA (1963), le rapport d'amplitude des ondes. S et des ondes $\mathrm{P}$ pour des ruptures de traction varie en fonction de l'azimut du plan de rupture par rapport à la direction source-capteur mais qu'il reste toujours inférieur à 4 .

La deuxième source possible de vibration du fait de l'injection de fluide dans un massif rocheux provient des micro-glissements induits par les variations de pression interstitielle (BLAIR et al., 1976; PEARSON, 1981). Depuis longtemps les sismologues êtudient le champ des vibrations associées à un relâchement de la contrainte de cisaillement le long de surfaces planes et de nombreuses informations sont maintenant disponibles à ce sujet notamment en ce qui concerne le spectre de Fourier de l'amplitude des déplacements pour les ondes $\mathrm{P}$ et pour les ondes $\mathrm{S}$ (KEILISBOROK, 1960; BRUNE, 1970; MADARIAGA, 1983). Nous nous contenterons de rappeler un certain nombre de résultats sans fournir les démonstrations correspondantes.

Dans le domaine des basses fréquences, l'amplitude des spectres des déplacements, tant pour les ondes P que pour les ondes $\mathrm{S}$, est constante, proportionnelle au moment sisimique $M_{0}$ et inversement proportionnelle au module de cisaillement dynamique du matériau $\mu$.
Rappelons que le moment sismique associé à un déplacement instantané $\underline{\mathrm{u}}$, uniforme, le long d'une surface plane d'aire $\mathrm{A}$ est égal à $\mu \mathrm{u} \mathrm{A}$; il caractérise l'intensité de l'événement. Considérant le spectre des ondes S, si $\Omega_{0}$ (s) est l'amplitude du plateau observé, KEILIS-BOROK (1960) a montré que:

$$
M_{o}=4 \pi \rho R \beta^{3} \frac{\Omega_{o}(s)}{R_{\theta \phi}(s)}
$$

où

$$
\begin{aligned}
& \rho \text { est la densité du matériau; } \\
& \mathrm{R} \quad \text { est la distance source-capteur: } \\
& \beta \\
& \mathrm{R}_{0 \phi}(\mathrm{s}) \text { est la vitesse des ondes } \mathrm{S} \text {; } \\
& \text { gramme de radiation de l'onde } \mathrm{S} \text {. }
\end{aligned}
$$

Dans le domaine des hautes fréquences, l'amplitude du spectre oscille; les maxima décroissant selon une puissance négative de la fréquence.

BRUNE (1970) a montré que pour ce type de rupture, la fréquence à partir de laquelle apparaît la décroissance de l'amplitude, appelée fréquence coin, dépend des dimensions de la source et de l'orientation de la dislocation vis-â-vis du point d'observation. HANKS et WYSS, 1972 ont montré que pour ce modèle, des résultats comparables pouvaient être obtenus pour l'onde $\mathrm{P}$ (fig. 5).

Depuis, de nombreux auteurs (voir par exemple SAVAGE, 1974; MADARIAGA, 1976 ; HARTZELL et BRUNE, 1977) se sont intéressés à la relation qui existe entre les dimensions de la source et la fréquence coin. Ils ont ainsi mis en évidence la dépendance de cette relation vis-à-vis du modèle interprétatif. BRUNE et al. en 1979, dans une étude comparée des différents modèles concluent que cette relation peut s'exprimer par:

$$
\mathrm{r}=\mathrm{K} \beta / \mathrm{f}_{\mathrm{e}}
$$

où $r$ est le rayon de la source supposée circulaire,

$\beta$ est la vitesse des ondes $S$,

$f_{c}$ est la fréquence coin pour le spectre des ondes $\mathrm{S}$,

$K$ est un facteur de proportionnalité.

$K$ dépend du type de rupture et de la position du point d'observation vis-à-vis de la dislocation. Ces auteurs concluent que $\mathrm{K}$ peut varier de 0,2 à 0,5 . Ils proposent de retenir la valeur moyenne de 0,33 indépendamment du modèle retenu.

Un dernier paramètre important peut être calculé à partir de la connaissance du moment sismique et de la géométrie de la source à savoir la chute de contrainte associée à la rupture. L'estimation de ce dernier paramètre dépend lui aussi des hypothèses faites sur la source (voir par exemple MADARIAGA, 1979). Dans le cas d'une fracture circulaire donnant lieu à une nupture uniforme sur toute sa surface, MADARIAGA propose la relation suivante:

$$
\Delta \sigma=\frac{M_{\circ}}{0,73 \pi R^{3}}
$$


où $\mathrm{R}$ est le rayon de la source. Il est donc possible de définir trois scalaires caractérisant approximativement la source:

- le moment sismique $\mathrm{M}_{\mathrm{o}}$ calculé à partir du plateau basse fréquence du spectre des déplacements;

- le rayon moyen de la source calculé à partir de la fréquence coin du spectre de l'amplitude des déplacements :

- la chute de contrainte moyenne associée à la dislocation calculée à partir du moment sismique et du rayon de la source.

On remarquera que le rayon de la source n'est connu qu'à un facteur deux près étant donné la grande dépendance de la fréquence coin vis-à-vis du modèle de source retenu. De ce fait la détermination de la chute de contrainte, qui en elle-même représente déjà une valeur moyennée sur la surface de glissement, n'est que très approximative puisqu'elle varie comme l'inverse du cube du rayon de la source.

\section{Résultats obtenus au Mayet-de-Montagne}

L'étude systématique des spectres fréquentiels du déplacement observé pour le bruit de fond durant une pêriode précédant chaque événement, puis pour les ondes $\mathrm{P}$ et pour les ondes $\mathrm{S}$ permet de mettre en évidence que l'énergie des signaux observés s'étale sur une large bande de fréquence (de 0,4 à $5 \mathrm{kHz}$ ) mais qu'elle ne sort de celle du bruit de fond qu'audessus de $400 \mathrm{~Hz}$, ce qui explique pourquoi aucun signal n'a été vu sur la sonde $0-250 \mathrm{~Hz}$ placée à $50 \mathrm{~m}$ de profondeur.

D'autre part les spectres du déplacement observé pour les ondes $\mathrm{P}$ et pour les ondes $\mathrm{S}$ présentent une portion basse fréquence relativement plate puis une portion descendante pour les fréquences plus élevées (voir l'exemple présenté sur la figure 5). Ces résultats suggèrent donc que les sources des événements observés sont associés à des microglissements et non à des mouvements d'ouverture.

Bien que l'estimation des paramètres caractérisant la source soit approximative, elle présente l'intérêt de fournir des ordres de grandeur représentatifs. Ainsi après avoir êtudié quelques signaux dans le détail, nous obtenons les valeurs suivantes:

$$
\begin{gathered}
\text { fréquence coin } \mathrm{f}_{\mathrm{c}}=1200 \mathrm{~Hz} \\
\text { moment sismique } \mathrm{M}_{\mathrm{o}}=11000 \mathrm{~N} . \mathrm{m} .
\end{gathered}
$$

Sachant que la vitesse des ondes S est de l'ordre de $3200 \mathrm{~m} / \mathrm{s}$ on en déduit que le rayon des sources est de l'ordre de $0,9 \mathrm{~m}$ et que la chute de contrainte est de Pordre de $5 \mathrm{kPa}$. Ces valeurs sont à rapprocher des résultats obtenus lors des essais de fracturation hydraulique entrepris à Los Alamos (PEARSON, 1981): pour des débits d'injection de $1,4 \mathrm{~m}^{3} / \mathrm{min}$ à une profondeur de $3000 \mathrm{~m}$, les chutes de contrainte moyennes observées ont varié de $50 \mathrm{kPa}$ à $180 \mathrm{kPa}$ et le rayon moyen des sources de $3,4 \mathrm{~m}$ à $2,1 \mathrm{~m}$, les fréquences coins varient de $0,3 \mathrm{kHz}$ à $1,4 \mathrm{kHz}$ valeurs qui ont été obtenues sans correction des effets de l'atténuation.

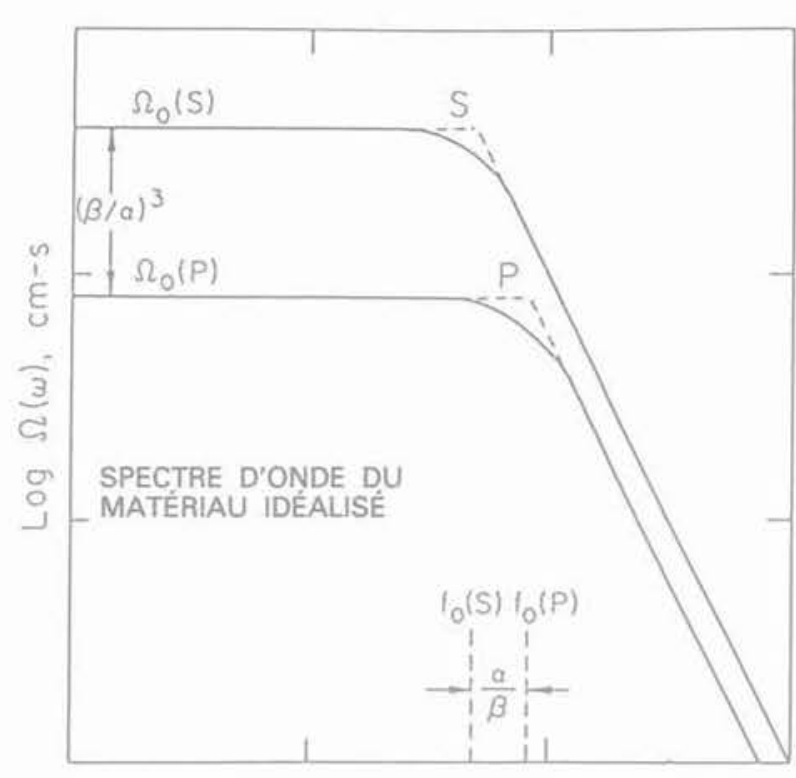

LOG DE LA FRÉQUENCE, $\mathrm{Hz}$

Fig. 5. - Spectres des ondes de volume selon le modèle de Brune (d'après HANKS et WYSS, 1972).

\section{DISCUSSION}

DROGUE et al. (1979) ont identifié, à partir d'études d'affleurements de granites proches du site des essais, quatre familles de fractures à pendange sub-vertical (entre $70^{\circ}$ et $90^{\circ}$ ) dans des directions $\mathrm{N} 20^{\circ} \pm 20^{\circ} \mathrm{E}$, $\mathrm{N} 60^{\circ} \pm 10^{\circ} \mathrm{E}, \mathrm{N} 100^{\circ} \pm 10^{\circ} \mathrm{E}$ et $\mathrm{N} 155^{\circ} \pm 5^{\circ} \mathrm{E}$ ainsi que des fractures sub-horizontales (pendages compris entre 0 et $20^{\circ}$ ), la densité de fractures subverticales dans une direction donnée dans un plan horizontal étant de une à deux fractures par mètre. Les observations faites avec une carnéra de forage ont permis de vérifier que ces cinq familles de fractures étaient également présentes en profondeur, au moins jusqu'à $250 \mathrm{~m}$.

Des essais de fracturation hydraulique entrepris pour la détermination des contraintes régionales ont conduit à l'ouverture de fractures sub-verticales dans les directions $\mathrm{N} 27^{\circ} \pm 2^{\circ} \mathrm{E}, \mathrm{N} 62^{\circ} \pm 7^{\circ} \mathrm{E}$ et $\mathrm{N} 155^{\circ} \pm 5^{\circ} \mathrm{E}$, directions qui appartiennent toutes aux familles de fractures observées en surface. La détermination du tenseur des contraintes régionales à partir de la mesure de la contrainte normale supportée par ces fractures a fourni les résultats indiqués dans le tableau II (CORNET et VALETTE, 1984). Un gradient de $26,5 \mathrm{kPa}$ $\mathrm{m}$ a été supposé pour la contrainte verticale. 


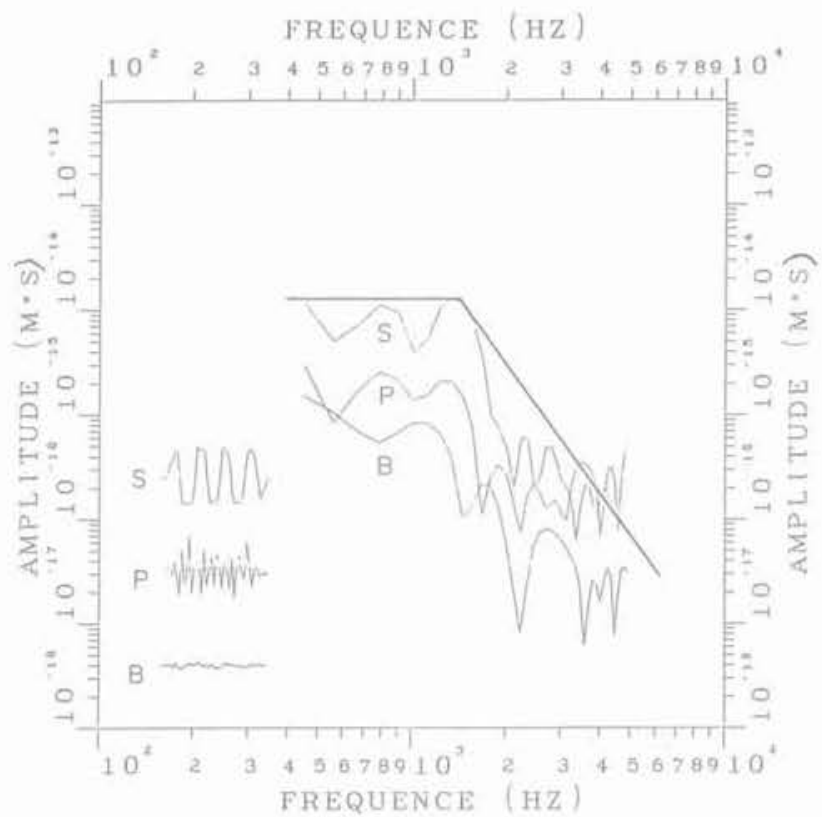

Fig. 6. - Exemple de spectres de déplacement observés pour le bruit de fond $(B)$, les ondes $P$ et les ondes $S$ d'un signal sismo-acoustique. Le signal pris en compte pour l'analyse est indiqué en bas à gauche. La durée du signal pour chacun de ces calculs était d'environ $3 \mathrm{~ms}$

Tableau 11. - Résultats des mesures de contrainte régionale; $z$ est la profondeur en mètres: $\sigma_{t}$ est la contrainte principale maximum horizontale (en MPa): $\sigma_{h}$ est la contrainte principale minimum horizontale (en MPa); $\lambda$ est la direction de $a_{H}$ et les $\epsilon$ sont les écarts-types des déterminations (d'après CORNET et VALETTE; 1984)

\begin{tabular}{|c|c|c|c|c|c|c|}
\hline $\mathrm{en}^{\mathrm{Z}} \mathrm{m}$ & $\begin{array}{c}\sigma_{\mathrm{H}} \\
\text { en } \\
\mathrm{MPa}\end{array}$ & $\begin{array}{l}\varepsilon \sigma_{\mathrm{H}} \\
e n \\
\mathrm{MPa}\end{array}$ & $\begin{array}{c}\sigma_{\mathrm{h}} \\
\text { en } \\
\mathrm{MPa}\end{array}$ & $\begin{array}{c}\varepsilon \sigma_{h} \\
\text { en } \\
\mathrm{MPa}\end{array}$ & $\lambda$ & $E \lambda$ \\
\hline $\begin{array}{r}50 \\
100 \\
150 \\
200\end{array}$ & $\begin{array}{l}7,1 \\
7,1 \\
7,1 \\
7,1\end{array}$ & $\begin{array}{l}1,5 \\
0,7 \\
0,6 \\
1,4\end{array}$ & $\begin{array}{l}1,2 \\
2,3 \\
3,5 \\
4,6\end{array}$ & $\begin{array}{l}0,8 \\
0,2 \\
0,4 \\
0,9\end{array}$ & $\begin{array}{lll}N & 16^{\circ} & E \\
N & 17^{\circ} & E \\
N & 17^{\circ} & E \\
N & 19^{\circ} & E\end{array}$ & $\begin{array}{l}5 \\
3 \\
3 \\
7\end{array}$ \\
\hline
\end{tabular}

La forme des signaux, notamment la présence d'ondes $\mathrm{S}$ bien marquées, et surtout les spectres de ces dernières permettent de conclure que ces événements ont été engendrés par des micro-glissements et non par des ouvertures de fractures hydrauliques.

Nous avons déterminé la contrainte normale $\left(\sigma_{N}\right)$ et tangentielle $\left(\sigma_{T}\right)$ ainsi que leur rapport $\left(\sigma_{T} / \sigma_{N}\right)$ à une profondeur de $200 \mathrm{~m}\left(\sigma_{\mathrm{H}}=7,1 \mathrm{MPa}, \sigma_{\mathrm{v}}=5,3 \mathrm{MPa}\right.$, $\sigma_{\mathrm{h}}=4,6 \mathrm{MPa}$ et $\beta=19^{\circ}$ ) d'une part pour les plans $1,2,3$ et 4 qui sont les plans, déterminés par moindres carrés, passant par les hypocentres des événements pour chaque période étudièe, et d'autre part pour les plans, a, b, c et d qui sont les quatre farnilles de fractures naturelles sub-verticales, $\mathrm{N} 20^{\circ} \pm$ $20^{\circ} \mathrm{E}, \mathrm{N} 60^{\circ} \pm 10^{\circ} \mathrm{E}, \mathrm{N} 100^{\circ} \pm 10^{\circ} \mathrm{E}$ et $\mathrm{N} 155^{\circ} \pm$ $5^{\circ} \mathrm{E}$ en supposant un pendage de $70^{\circ}$ (tableau III). La comparaison des rapports $\sigma_{\mathrm{T}} / \sigma_{\mathrm{N}}$ montre que les plans de fracturation naturelle $\mathrm{b}$ et $\mathrm{d}$ sont plus susceptibles de donner lieu à des ruptures par cisaillement que les autres plans.

Tableau III. - Les contraintes normales, tangentielles et leur rapport pour les plans définis par moindres carrés et pour les plans de fractures naturelles, (en $\mathrm{MPa}$ )

\begin{tabular}{|l|c|c|c|c|c|c|c|c|}
\hline & \multicolumn{3}{|c|}{$\begin{array}{c}\text { Plans passant } \\
\text { par les } \\
\text { hypocentres }\end{array}$} & \multicolumn{4}{c|}{$\begin{array}{c}\text { Plans } \\
\text { de fracturation } \\
\text { naturelle }\end{array}$} \\
\cline { 2 - 9 } & 1 & 2 & 3 & 4 & a & b & c & d \\
\hline$\sigma_{N}$ & 5,73 & 5,37 & 5,35 & 5,52 & 4,68 & 5,63 & 6,84 & 5,75 \\
$\sigma_{T}$ & 0,79 & 0,43 & 0,35 & 0,59 & 0,23 & 1,17 & 0,67 & 1,19 \\
$\sigma_{T} / \sigma_{N}$ & 0,14 & 0,08 & 0,07 & 0,11 & 0,05 & 0,21 & 0,10 & 0,21 \\
\hline
\end{tabular}

Ces déterminations sont représentées de façon synthétique dans une représentation de Mohr sur la figure 7. Celle-ci suggère qu'une augmentation de la pression interstitielle de lordre de 3,8 $\mathrm{MPa}$ dans le massif pourrait être la cause de ces micro-ruptures, si l'on suppose que la résistance au cisaillement des fractures préexistantes satisfait le critère de Coulomb en retenant une cohésion nulle:

$$
|\tau| \leqslant \mu .\left(\sigma_{N}-P\right)
$$

Ce critère est représenté sur la figure 7 pour deux valeurs du coefficient de frottement: $\mu=0,5$ et $\mu=0,7$. PEARSON (1981) propose une valeur de 0,58 pour ce coefficient étant donné que l'angle de frottement des surfaces de fracture du granite est de l'ordre de $30^{\circ}$.

Notons que l'augmentation de pression nécessaire pour provoquer des micro-glissements est inférieure à la valeur requise pour propager de vraies fractures hydrauliques, à savoir des fractures normales à la contrainte principale minimum, qui est de $4,6 \mathrm{MPa}$.

Ces résultats suggèrent donc que la distribution des hypocentres dans une zone sub-horizontale est liée à la présence d'une zone de forte conductivité hydraulique préexistante, mais que l'activité sismo-acoustique proprement dite serait liée à la percolation du fluide le long de fractures sub-verticales naturelles recoupant les épontes de la zone conductrice. Cette interprétation serait ainsi en accord avec le fait que les événements semblent s'éloigner du plan de forte conductivité hydraulique au cours du temps. 


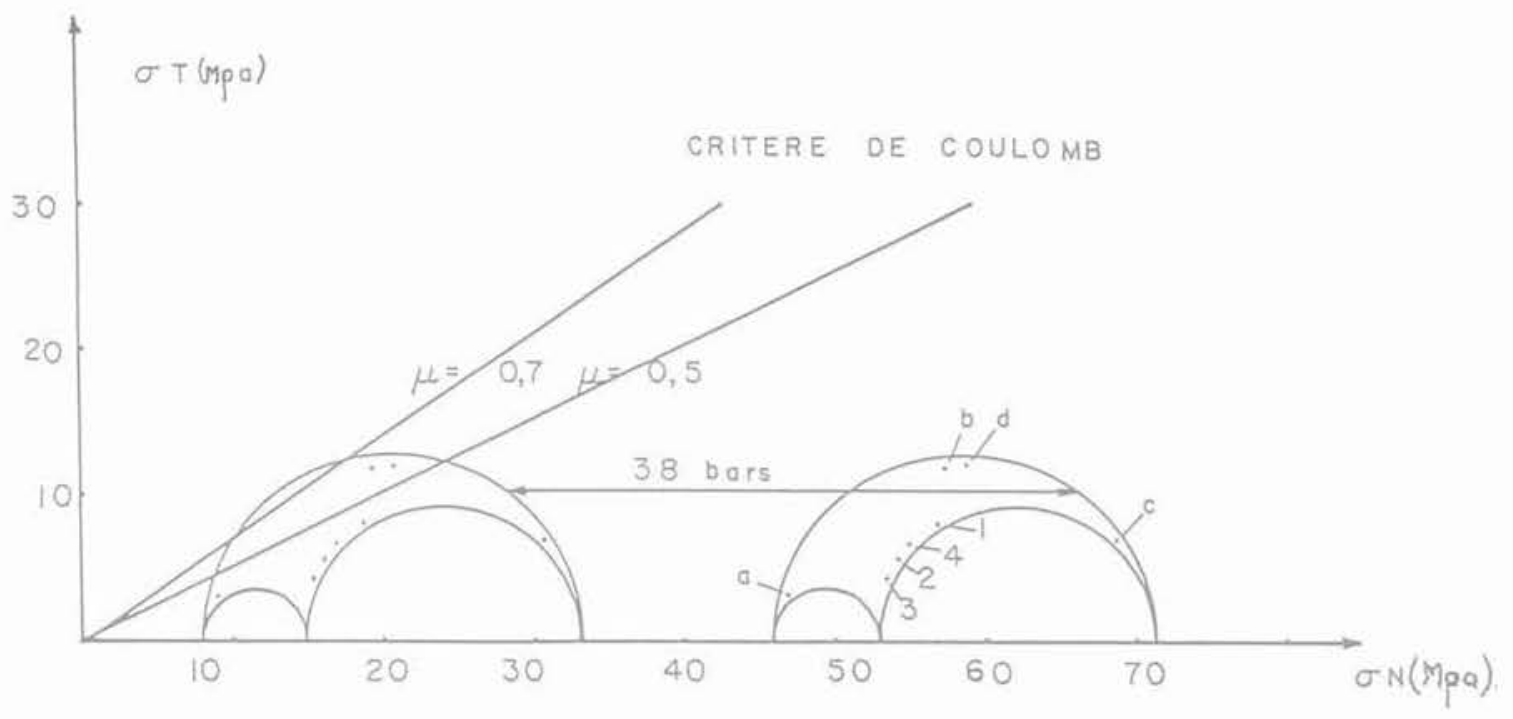

Fig. 7. - Représentation de Mohr du champ de contrainte. Les contraintes normales et tangentielles pour les plans (1) à (4) et (a) à (d), avant et après une augmentation de 3,8 MPa de la pression interstitielle sont également indiquées.

\section{CONCLUSIONS}

Le système d'acquisition et de numérisation des données pour une bande de fréquence $100-3000 \mathrm{~Hz}$, s'est révélé a posteriori adéquate pour l'étude de l'activité sismo-acoustique engendrée par l'injection de gel à $200 \mathrm{~m}$ de profondeur dans le massif granitique du Mayet-de-Montagne.

Les hypocentres des événements, déterminés à partir des lectures de temps d'arrivée des ondes $\mathrm{P}$ et $\mathrm{S}$, ont tendance à migrer vers le puits III-3. Ceci est en parfait accord avec la montée de $2 \mathrm{~m}$ du niveau d'eau dans ce forage constatée sur le terrain.

D'autre part, les plans, déterminés par moindres carrés passant par les hypocentres observés durant diverses périodes de pompage, sont de pendages plutôt faibles (14 à $31^{\circ}$ ), ce qui indique qu'une zone plutôt horizontale du massif a été stimulée. De plus, la distance moyenne entre le point d'injection et les hypocentres augmentent au cours du temps, ce qui indique une diffusion progressive du fluide dans le massif.

Une analyse spectrale des signaux suggère que la source de ces événements a très probablement correspondu à des glissements suivant des plans de fractures préexistantes. Elle fournit des valeurs de fréquence-coins de l'ordre de $1200 \mathrm{~Hz}$, des moments sismiques de l'ordre de $11000 \mathrm{~N} . \mathrm{m}$., des rayons de surface de glissement de l'ordre de $0,9 \mathrm{~m}$ pour des sources circulaires, et des chutes de contraintes de l'ordre de $5 \mathrm{kPa}$, si l'on ne tient pas compte des effets de l'atténuation

L'analyse du champ de contraintes dans le massif montre que des augmentations de pression interstitielle de l'ordre de $3,8 \mathrm{MPa}$ devraient suffire à provoquer la rupture par glissement suivant les fractures naturelles préexistantes sub-verticales qui recoupent les épontes de la zone conductrice horizontale.

\section{REMERCIEMENTS}

Nous tenons à remercier $M$. Gunter LEYDECKER du * Bundesanstalt für Geowissenschaften und Rohstoffe (B.G.R.) = pour sa participation aux essais, ainsi que M. Louis MARTEL de "L'Institut de Physique du Globe de Paris (I.P.G.P.) , pour sa collaboration durant les traitements préliminaires.

Nous remercions également * L'Institut National d'Astronomie et de Géophysique (I.N.A.G.) * et en particulier Madame Danielle FOUASSIER pour la numérisation des signaux.

S. TALEBI a bénéficié au cours de ce travail d'une bourse de "L'Institut Français du pétrole (I.F.P.) \$. Ces essais ont été financés par «La Communauté Economique Européenne, contrat CEE-EGP-1-002F.

\section{BIBLIOGRAPHIE}

ALBRIGHT J.N., PEARSON C.F. (1980). - * Location of hydraulic fractures using microseismic techniques *. SPE paper 9509, 55 th Annual Fall Technical Conference and Exhibition of the Society of Petroleum Engineers, Dallas, Texas.

AKI K., FEHLER M., DAS S. (1977). - « Source mechanism of volcanic tremor: fluid driven crack models and their application to the 1963 Kilauea eruption *. Joumal of volcanology and Geothermal Research, vol. 2, pp. 259-287. 
AUDRIC J. (1980). - * Etude in situ des propriétés acoustiques du granite du Mayet-de-Montagne ". Thèse de $3^{e}$ cycle, Univ. Paris VI.

BLAIR A.G., TESTER J.W., MORTENSEN J.J. (1976). - "Hot dry rock geothermal project». Rep. LA 6525, Los Alamos Scientific laboratories, New Mexico, U.S.A.

BRUNE J.N. (1970). - « Tectonic stress and the spectra of seismic shear waves from earthquakes:. J. Geophys. Res., vol. 75, nb 26, pp. 4997-5009.

BRUNE J.N., ARCHULETA R.J., HARTZELL S. (1979). - a Far field S-wave spectra comer frequencies and pulse shapes ». J. Geophys. Res., vol. 84, nb B5, pp. 2262-2272.

CHOUET B. (1981). - " Ground motion in the near field of a fluid driven crack and its intepretation in the study of shallow volcanic Tremor $\$ . J$. Geophys. Res., vol. 86, nb B7, pp. 5985-6016.

CORNET F.H., VALETTE B. (1984). - - In situ stress detemination from hydraulic injection test data . J. Geophys, Res., vol. 89, nB13, pp. 11527. 11537.

DROGUE C., GRILLOT J.C., RAZACK M. (1979). "Site du Mayet-de-Montagne: Etude de la fracturation ", Rapport interne, I.N.A.G., Paris.

HANKS T.C., WYSS M. (1972). - « The use of bodywave spectra in the determination of seismic source parameters $\star$. Bulletin of seismological Society of America, vol. 62, n², pp. 561-589.

HARTZELL S.H., BRUNE J.N. (1977). - « Source parameters for the january, 1975, Brawley imperial valley earthquake swarm *; Pure and Appl. Geophys., vol. 115 , pp. 333-335.

KEILIS-BOROK V.1. (1960). - "Investigation of the Mechanism of Earthquakes *. Sov. Res. Geophys. 4 (Engl. Tr. Geofiz. Inst., vol. 40, 1957), 201 pp. Am. Geophys. Union, Consultants Bureau, N.Y.

LEYDECKER G. (1981). - " Seismische ortung hydraulish erzeugter Brüche im Geothermik Frac Projekt Falkenberg ». Bundesanstalt für Geowissenshaften und Rohstoffe, Archiv-Nr. 86 549, 104 pages, Hanover, R.F.A.
LEYDECKER G. (1982). - « Seismische ortung hydraulish erzeugter Brüche im französischen Geothermik projekt Le Mayet ». Bundensanstalt für Geowissenschaften und Rohstoffe, Archiv-Nr 92 297, 25 pages, Hannover, R.F.A.

MADARIAGA R. (1976). - "Dynamics of an expanding circular fault ». Bull. Seism. Soc. Am., vol. 66 , pp. $636-666$.

MADARIAGA R. (1979). - "On the relation between Seismic Moment and stress drop in the presence of stress and strenght heterogeneity s. J. Geophys. Res., vol. 84, B5, pp. 2243-2250.

MADARIAGA R. (1983). - «Earthquake Source theory, a review in earthquakes: observation, theory and interpretation . LXXXV Corso, Soc. Italiana di Fisica, pp. 1-44, Bologna, Italy.

POWER D.V., SCHUSTER C.L., HAY R. (1975). J. Petrol. Tech., paper SPE 5626.

PEARSON C. (1981). - «The relationship between Microseismicity and High Pore Pressures during hydraulic stimulation experiments in low permeability granitic rocks s. J. Geophys. Res., vol. 86, nb B9, pp. 7855-7864.

RICE J. (1980). - * The mechanics of earthquake rupture ; in Physics of the Earth interior *. LXXVIII Corso, Societa Italiana di Fisica, Bologna, Italy, pp. 555-649.

RUMMEL F. (1985). - *Fracture Mechanics Approach to Hydraulic Fracturing Stress measurements: Rock fracture Mechanics, B. Atkinson ed., Academic Press, London.

SAVAGE J.C. (1974). - «Relation between $P$ and $S$. wave comer frequencies in the seismic spectrum ». Bull. Seis. Soc. Am., vol. 64, pp. 1621-1627.

SAVAGE J.C., MANSINHA L. (1963). - - Radiation from a tensile fracture \. J. Geophys. Res., vol. 68, $n^{\circ} 23$, pp. 6345-6358.

ZOBACK M.D., POLLARD D.D. (1978). $-*$ Hydraulic fracture propagation and the interpretation of pressure time records for in situ stress determination „. Proc. U.S. Symp. Rock Mech, 19 th, pp. 14-22. 
